\title{
Construction of a Predictive Score for Hemodialysis in Acute Theophylline Intoxicated Patients
}

\author{
Wesam Abd El-Salam Abd El-Wahab ${ }^{1 *}$; Rania Hussien ${ }^{2}$
}

\begin{tabular}{|c|c|}
\hline & ABSTRACT \\
\hline $\begin{array}{l}\text { KEYWORDS } \\
\text { Theophylline, } \\
\text { Poisoning, } \\
\text { Predictive score, } \\
\text { Hemodialysis. }\end{array}$ & $\begin{array}{l}\text { Theophylline toxicity could be life-threatening due to refractory } \\
\text { hypotension, severe cardiac arrhythmias or convulsions that may require } \\
\text { extracorporeal removal of theophylline via hemodialysis. This study aimed to } \\
\text { evaluate theophylline toxic manifestations to construct a predictive score to } \\
\text { identify patients who are at risk and require hemodialysis. This study was a cross } \\
\text { sectional comparative study included patients with acute theophylline toxicity } \\
\text { admitted to the Poison Control Center of Ain Shams University Hospitals during } \\
\text { six months from the first of January } 2019 \text { to the end of June } 2019 \text {. Collected data } \\
\text { included sociodemographic, intoxication and clinical data. Outcome of patients and } \\
\text { their needs for hemodialysis were also recorded. Routine investigations and } \\
\text { theophylline levels were done for each patient in addition to ECG recordings. This } \\
\text { study enrolled } 175 \text { theophylline intoxicated patients who met the inclusion criteria. } \\
\text { All studied patients ingested theophylline intentionally, most of them were females } \\
\text { with mean age } 22.11 \pm 8.65 \text { years. The mean delayed time was } 7.29 \pm 5.08 \text { hours. } \\
\text { Nausea and vomiting were the most common clinical manifestations, } 4.6 \% \text { of } \\
\text { studied patients needed hemodialysis and all cases were discharged with no deaths. } \\
\text { All cases were tachypneic, most of them were hypertensive, tachycardic and had } \\
\text { abnormal ECG findings. In conclusion; theophylline poisoning hemodialysis (TPH) } \\
\text { score was constructed to find out the probability of patients need for hemodialysis. } \\
\text { It consisted of nine important easily measured parameters which are theophylline } \\
\text { and HCO levels, duration of hospital stay, pulse, respiratory rate, presence of } \\
\text { hematemesis, seizures, agitation and abnormal ECG findings. Any case with } 5 \\
\text { changes or more is critical and may need hemodialysis. }\end{array}$ \\
\hline
\end{tabular}

\section{Introduction}

Theophylline; a natural ingredient of tea and cocoa plants, is widely used as bronchodilator for treatment of asthma and chronic obstructive pulmonary disease (COPD) in the developing countries due to its cheap

\footnotetext{
${ }^{(1)}$ Forensic Medicine and Clinical Toxicology Department- Faculty of Medicine for Girls-Al-Azhar University

(2) Forensic Medicine and Clinical Toxicology Department-Faculty of Medicine- Ain Shams University.

*The corresponding author: wabdalwahab@yahoo.com w_abdalwahab@azhar.edu.eg
}

price. Additionally, it is used as antiinflammatory, diuretic, smooth muscle relaxant, and also stimulant to respiratory, nervous and cardiac systems (Barnes, 2013; Wu et al., 2013).

It has multiple mechanisms of action as it inhibits phosphodiesterase enzyme, antagonizes adenosine receptors, enhances the release of endogenous catecholamines and stimulates beta-adrenergic receptors (Greene et al., 2018).

Theophylline is not prescribed as a firstline medication due to its narrow therapeutic index (Hopkins and MacKenzie-Ross, 2016), its toxicity is dose-related and most of its 
benefits occur only when near-toxic doses are used (Horita et al., 2016). Toxicity of theophylline occurs either acute that results from intake of high doses or chronic from overmedication (Yaman et al., 2016).

Symptoms of theophylline toxicity are non-specific as multiple organs may be affected and patient presented with various clinical manifestations such as nausea, vomiting, tachycardia, tremors, agitation, seizures and hypotension (Kapoor et al., 2015; Hopkins and MacKenzie-Ross, 2016). Some of these manifestations could be life threatening, due to refractory hypotension, severe cardiac arrhythmias or convulsions that may require extracorporeal removal of theophylline via hemodialysis (Barnes, 2013; Kapoor et al., 2015).

Early recognition of severity of theophylline toxicity can be lifesaving that needs aggressive supportive care to improve clinical outcomes of patients (Aggelopoulou et al., 2018). Therefore, this study aimed to evaluate theophylline toxic manifestations to construct a predictive score to identify patients who are at risk and require hemodialysis to improve management of acute theophylline intoxicated patients as early diagnosis and appropriate treatment is often lifesaving.

\section{Subjects and Methods}

\section{Type of the study}

A cross-sectional comparative study.

\section{Inclusion criteria}

This study included patients of both sexes with isolated acute theophylline toxicity who were admitted to the Poison Control Center of Ain Shams University Hospitals (PCC-ASUH) during a period of six months from the first of January 2019 to the end of June 2019.

\section{Exclusion criteria}

Patients with co administration of drugs or poisons and patients with history of chronic cardiac, respiratory, hepatic or renal diseases were excluded from the study.

\section{Ethical considerations}

The study was approved by the Research Ethics Committee of Faculty of medicine for girls Al-Azhar University with a code number (202001059) and a full informed consent was obtained from patients or their guardians.

\section{Data collection}

A special observation sheet was designed included demographic data (age, sex), intoxication data (theophylline dose, route of exposure, delay time, mode of poisoning, symptoms), in addition to vital data (pulse, blood pressure and respiratory rate). Normal values were stated according to McGrath and Bachmann (2018). Outcome of patients, need for mechanical ventilation, hemodialysis and duration of hospital stay were also recorded.

Neurological, cardiovascular and respiratory systems examinations were performed for each patient on admission. All patients received therapeutic interventions including gut decontamination, charcoal administration and standard supportive care when indicated following the guidelines of the PCC-ASUH protocols. Patients who presented with severe manifestations like respiratory distress, arrhythmia, shock, coma or convulsions were admitted to ICU.

\section{Sampling}

Two venous blood samples were collected on admission for each patient before receiving any treatment. One was 
anticoagulated with EDTA to measure total leukocytic count (TLC). The other sample was transferred to a clean dry centrifuge tube and left for few hours to clot. After complete clotting, it was centrifuged for 10 minutes at $5000 \mathrm{rpm}$. Serum was separated, stored in the freezer and used later after collection of all samples for measurement of serum glucose and $\mathrm{K}^{+}$. Serum theophylline level was measured according to Sheehan and Haythorn (1976). On the other hand, arterial blood sample was collected in heparinized syringes for immediate measurement of arterial blood gases.

\section{ECG analysis}

It included rate, rhythm, ST/T abnormalities and measurement of Q-T intervals. The QT interval was corrected (QTc) according to the formula of Bazett, in which the QT interval is adjusted for heart rate by dividing it by the square root of the R-R interval. $\mathrm{QTc}=\mathrm{QT} / \sqrt{\mathrm{RR}}$ (Postema and Wilde, 2014).

\section{Statistical analysis}

Recorded data were analyzed using the statistical package for social sciences, version 20.0 (SPSS Inc., Chicago, Illinois, USA). Quantitative data were expressed as mean \pm standard deviation (SD). Qualitative data were expressed as frequency and percentage. Independent-samples t-test of significance was used when comparing between two means. Mann Whitney z-test: for two-group comparisons in non-parametric data. Chisquare (x2) test of significance was used in order to compare proportions between qualitative parameters. The confidence interval was set to $95 \%$ and the margin of error accepted was set to $5 \%$. p value level of significance; $p>0.05$ : Non-significant. $p \leq 0.05$ : Significant. $p \leq 0.001$ : Highly significant.
Receiver operating characteristic (ROC curve) analysis was used to find out the overall predictivity of parameter in and to find out the best cut-off value with detection of sensitivity and specificity at this cut-off value.

\section{Results}

This study was conducted on 175 cases with acute theophylline toxicity were admitted to the PCC-ASUH during the study period and fulfilled inclusion and exclusion criteria.

Most of studied patients were females $157(89.7 \%)$ with the mean age of $22.11 \pm 8.65$ years. According to the mode of poisoning, it was suicidal in all cases. All patients had taken theophylline through ingestion with mean dose of $10.69 \pm 8.55$ tablets. The mean delayed time was 7.29 \pm 5.08 hours (Table 1).

Table (1): Descriptive parameters of the theophylline intoxicated patients admitted to the Poison Control Center of Ain Shams University Hospitals.

\begin{tabular}{|c|c|}
\hline Parameters & Total $(\mathbf{n = 1 7 5})$ \\
\hline $\begin{array}{c}\text { Sex } \\
\text { Female }\end{array}$ & $\begin{array}{c}18(10.3 \%) \\
157(89.7 \%)\end{array}$ \\
\hline $\begin{array}{c}\text { Age (years) } \\
\text { Range (Mean } \pm \text { SD) }\end{array}$ & $\begin{array}{c}12-60 \\
(22.11 \pm 8.65)\end{array}$ \\
\hline $\begin{array}{c}\text { Mode and route } \\
\text { Suicidal by oral route }\end{array}$ & $175(100.0 \%)$ \\
\hline $\begin{array}{c}\text { Delay time (hours) } \\
\text { Range (Mean } \pm \text { SD) }\end{array}$ & $1-24(7.29 \pm 5.08)$ \\
\hline $\begin{array}{c}\text { Amount (tablets) } \\
\text { Range (Mean } \pm \text { SD) }\end{array}$ & $2-40(10.69 \pm 8.55)$ \\
\hline
\end{tabular}

$\mathrm{n}=$ number SD: standard deviation

Table (2) shows the characteristic clinical presentations of studied patients. Nausea and vomiting were the most common symptoms of the theophylline poisoned cases (98.9\%), followed by abdominal pain in $41.1 \%$, agitation in $10.9 \%$ and hematemesis in $8.6 \%$ of cases, $1.1 \%$ of studied patients was shocked and $0.6 \%$ had seizures. 
Table (2): Characteristic clinical presentations of acute theophylline intoxicated patients admitted to the Poison Control Center of Ain Shams University Hospitals.

\begin{tabular}{|l|c|}
\hline \multicolumn{1}{|c|}{ Clinical presentations } & Total $(\mathbf{n}=\mathbf{1 7 5})$ \\
\hline Nausea \& Vomiting & $173(98.9 \%)$ \\
\hline Abdominal pain & $72(41.1 \%))$ \\
\hline Agitation & $19(10.9 \%)$ \\
\hline Hematemesis & $15(8.6 \%)$ \\
\hline Shock & $2(1.1 \%)$ \\
\hline Seizures & $1(0.6 \%)$ \\
\hline
\end{tabular}

$\mathrm{n}=$ number
According to vital data of the studied cases, there was a highly significant change $(\mathrm{p}$ $<0.001)$ of blood pressure in 44 cases $(25.1 \%)$, most of them (37 cases - 84.1\%) were hypertensive. More than half of patients $(57.7 \%)$ were tachycardic which was statistically significant as confirmed by Z-test. Tachypnea was found in all cases $(100 \%)$ (Table 3).

Table (3): Vital data of acute theophylline intoxicated patients admitted to the Poison Control Center of Ain Shams University Hospitals.

\begin{tabular}{|c|c|c|c|}
\hline Vital data & Total $(n=175)$ & z-test & p-value \\
\hline \multicolumn{4}{|l|}{ Pulse } \\
\hline Normal & $74(42.3 \%)$ & \multirow{3}{*}{139.121} & \multirow{3}{*}{$<0.001 * *$} \\
\hline Tachycardia & $101(57.7 \%)$ & & \\
\hline Range (Mean \pm SD) & $60-160(105.10 \pm 16.89)$ & & \\
\hline \multicolumn{4}{|l|}{ Blood pressure } \\
\hline Normal & $131(74.9 \%)$ & \multirow{3}{*}{29.536} & \multirow{3}{*}{$<0.001^{* *}$} \\
\hline Hypertension & $37(21.1 \%)$ & & \\
\hline Hypotension & $7(4 \%)$ & & \\
\hline \multicolumn{4}{|l|}{ Respiratory rate } \\
\hline Normal & $0(0 \%)$ & \multirow{3}{*}{346.011} & \multirow{3}{*}{$<0.001 * *$} \\
\hline Tachypnea & $175(100 \%)$ & & \\
\hline Range (Mean \pm SD) & $22-32(25.59 \pm 1.84)$ & & \\
\hline
\end{tabular}

$\mathrm{n}=$ number; SD: standard deviation, p-value $>0.05$ Non-Significant; *p-value $<0.05$ Significant; $* *$ p-value $<0.001$ highly significant.

Table (4) shows laboratory investigations of theophylline intoxicated patients; the mean theophylline level was $40.38 \pm 25.30$. All cases of the present study had high total leucocytic count (TLC), most of them were hyperglycemic (76\%), hypokalemic $(64.6 \%)$ and the blood $\mathrm{pH}$ was acidic in $66.7 \%$ of the cases. 
Table (4): Laboratory investigations of theophylline intoxicated patients admitted to the Poison Control Center of Ain Shams University Hospitals.

\begin{tabular}{|c|c|c|c|}
\hline Parameters & Total $(n=175)$ & z-test & p-value \\
\hline Theophylline level $(\mu \mathrm{g} / \mathrm{ml})$ Range (Mean \pm SD) & $15-90.8(40.38 \pm 25.30)$ & & \\
\hline \multicolumn{4}{|l|}{ Serum Glucose $(\mathrm{mg} / \mathrm{dl})$} \\
\hline Normal & $41(23.4 \%)$ & \multirow{4}{*}{214.032} & \multirow{4}{*}{$<0.001 * *$} \\
\hline Hyperglycemia & $133(76 \%)$ & & \\
\hline Hypoglycemia & $1(0.6 \%)$ & & \\
\hline Range (Mean \pm SD) & $66-340(151.60 \pm 53.66)$ & & \\
\hline \multicolumn{4}{|l|}{ Serum K (mg/dl) } \\
\hline Normal & $62(35.4 \%)$ & \multirow{3}{*}{164.046} & \multirow{3}{*}{$<0.001 * *$} \\
\hline Hypokalemia & $113(64.6 \%)$ & & \\
\hline Range (Mean \pm SD) & $1.9-4.8[3.21 \pm 0.59]$ & & \\
\hline \multicolumn{4}{|l|}{ TLC } \\
\hline Normal & $30(17.2 \%)$ & \multirow{3}{*}{203.021} & \multirow{3}{*}{$<0.001 * *$} \\
\hline Leukocytosis & $145(82.8 \%)$ & & \\
\hline Range $[$ Mean \pm SD] & $9.3-23.4(18.13 \pm 9.49)$ & & \\
\hline \multirow{2}{*}{\multicolumn{4}{|c|}{$\begin{array}{l}\text { Arterial blood gases } \\
\text { pH }\end{array}$}} \\
\hline & & & \\
\hline Normal & $91(52 \%)$ & \multirow{4}{*}{107.910} & \multirow{4}{*}{$<0.001 * *$} \\
\hline High & $28(16 \%)$ & & \\
\hline Low & $56(32 \%)$ & & \\
\hline Range (Mean \pm SD) & $7.37-7.58(7.37 \pm 0.24)$ & & \\
\hline \multicolumn{4}{|l|}{ PCO2 $(\mathrm{mmHg})$} \\
\hline Normal & $92(52.6 \%)$ & \multirow{4}{*}{106.111} & \multirow{4}{*}{$<0.001 * *$} \\
\hline High & $15(8.6 \%)$ & & \\
\hline Low & $68(38.9 \%)$ & & \\
\hline Range (Mean \pm SD) & $13-57(35.33 \pm 7.82)$ & & \\
\hline \multicolumn{4}{|l|}{ HCO3 $(\mathrm{mmol} / \mathrm{l})$} \\
\hline Normal & $77(44.0 \%)$ & \multirow{4}{*}{133.348} & \multirow{4}{*}{$<0.001^{* *}$} \\
\hline Low & $94(53.7 \%)$ & & \\
\hline High & $4(2.3 \%)$ & & \\
\hline Range (Mean \pm SD) & $9.3-38.5(21.32 \pm 3.97)$ & & \\
\hline
\end{tabular}

$\mathrm{n}=$ number; TLC: total leucocytic count SD: standard deviation, $\mathrm{p}$-value $>0.05$ : non-significant; *p-value $<0.05$ : significant; **pvalue $<0.001$ : highly significant.

Regarding ECG abnormalities among studied patients, most of cases (57.7\%) had abnormal ECG findings which were statistically significant as confirmed by Z-test. Theophylline had high significant effect on the heart rate, QTc interval and $\mathrm{T}$ wave. The most common ECG abnormality was sinus tachycardia in $57.7 \%$ followed by prolonged QTc interval in $8.0 \%$ and abnormal $\mathrm{T}$ wave in $6.3 \%$ of the cases (Table 5 ). 
Table (5): Electrocardiographic (ECG) abnormalities of acute theophylline intoxicated patients admitted to the Poison Control Center of Ain Shams University Hospitals.

\begin{tabular}{|c|c|c|c|}
\hline Parameters & $\begin{array}{c}\text { Number } \\
(\%)\end{array}$ & z-test & p-value \\
\hline $\begin{array}{c}\text { ECG comment } \\
\text { Normal } \\
\text { Abnormal }\end{array}$ & $\begin{array}{c}74(42.3 \%) \\
101(57.7 \%)\end{array}$ & 139.121 & $<0.001^{* *}$ \\
\hline $\begin{array}{c}\text { Rate } \\
\text { Sinus } \\
\text { tachycardia }\end{array}$ & $101(57.7 \%)$ & 11.58 & $0.001^{*}$ \\
\hline $\begin{array}{c}\text { QTe interval } \\
\text { Normal } \\
\text { Prolonged }\end{array}$ & $161(92.0 \%)$ \\
\hline $\begin{array}{c}\text { T wave } \\
\text { Normal } \\
\text { Abnormal }\end{array}$ & $164(9.0 \%)$ & 12.574 & $0.004^{*}$ \\
\hline $\begin{array}{c}\text { ST segment } \\
\text { Normal } \\
\text { Abnormal }\end{array}$ & $11(6.3 \%)$ & 9.412 & $0.002^{*}$ \\
\hline
\end{tabular}

p-value $>0.05$ Non significant; *p-value $<0.05$ Significant; $* *$ p-value $<0.001$ highly significant.

Regarding the outcome of studied patients; most of them were admitted to the inpatient unit $(95.4 \%)$ and 8 cases $(4.6 \%)$ only were admitted to ICU and all studied cases discharged with no deaths. The mean duration of hospital stay was $1.57 \pm 0.72$ days. Eight cases $(4.6 \%)$ needed hemodialysis and none of patients needed mechanical ventilation (Table 6).

Table (6): Outcome of acute theophylline intoxicated patients admitted to the Poison Control Center of Ain Shams University Hospitals

\begin{tabular}{|l|c|}
\hline \multicolumn{1}{|c|}{ Outcome } & $\begin{array}{c}\text { Total } \\
(\mathbf{n = 1 7 5 )}\end{array}$ \\
\hline Admission to the inpatient unit & $167(95.4 \%)$ \\
\hline Admission to ICU & $8(4.6 \%)$ \\
\hline $\begin{array}{l}\text { Duration of hospital stay (day) } \\
\text { Range (Mean } \pm \text { SD) }\end{array}$ & $\begin{array}{c}1-5 \\
(1.57 \pm 0.72)\end{array}$ \\
\hline Hemodialysis & $8(4.6 \%)$ \\
\hline Need mechanical ventilation & $0(0 \%)$ \\
\hline Deaths & $0(0 \%)$ \\
\hline
\end{tabular}

$\mathrm{n}=$ number; SD: standard deviation ICU: intensive care unit,

In the current study, a comparison was done between cases who needed hemodialysis and cases who did not according to all studied parameters to construct a predictive score to identify those who were at risk and required hemodialysis. There was a significant difference between them in nine parameters which were: theophylline level, duration of hospital stay, respiratory rate, pulse, $\mathrm{HCO} 3$, presence of hematemesis, seizures, agitation and ECG abnormalities (Tables 7-10).

Table (7): Comparison between patients who needed hemodialysis and patients who did not need according to descriptive parameters.

\begin{tabular}{|l|c|c|c|c|}
\hline \multicolumn{1}{|c|}{ Parameters } & \multicolumn{2}{c|}{ Hemodialysis } & \multirow{2}{*}{ x2/t-test } & \multirow{2}{*}{ p-value } \\
\cline { 2 - 3 } & $\begin{array}{c}\text { No } \\
(\mathbf{n = 1 6 7 )}\end{array}$ & $\begin{array}{c}\text { Yes } \\
(\mathbf{n}=\mathbf{8})\end{array}$ & & \\
\hline Sex & $16(9.6 \%)$ & $2(25.0 \%)$ & 1.967 & 0.161 \\
$\quad$ Male & $151(90.4 \%)$ & $6(75.0 \%)$ & & \\
$\quad$ Female & $22.15 \pm 8.78$ & $21.25 \pm 5.47$ & 0.082 & 0.775 \\
\hline Age (years) (Mean \pm SD) & $7.37 \pm 5.12$ & $5.63 \pm 4.1$ & 0.900 & 0.344 \\
\hline Delay time (hours) (Mean \pm SD) & $10.68 \pm 8.57$ & $10.86 \pm 8.88$ & 0.003 & 0.957 \\
\hline Amount (tablets) (Mean \pm SD) & $1.53 \pm 0.69$ & $2.38 \pm 0.74$ & 11.375 & $<0.001^{* *}$ \\
\hline Time of hospital stay (days) (Mean \pm SD)
\end{tabular}

$\mathrm{n}=$ number; SD standard deviation; t-Independent Sample t-test; $\mathrm{x} 2$ : Chi-square test p-value $>0.05$ Non significant; ${ }^{*} \mathrm{p}$-value $<0.05$ Significant; $* *$ p-value $<0.001$ highly significant. 
Table (8): Comparison between patients who needed hemodialysis and patients who did not need according to clinical presentations.

\begin{tabular}{|c|c|c|c|c|}
\hline \multirow[b]{2}{*}{ Parameters } & \multicolumn{2}{|c|}{ Hemodialysis } & \multirow[b]{2}{*}{ x2/t-test } & \multirow[b]{2}{*}{ p-value } \\
\hline & $\begin{array}{c}\text { No } \\
(n=167)\end{array}$ & $\begin{array}{c}\text { Yes } \\
(n=8)\end{array}$ & & \\
\hline $\begin{array}{c}\text { Nausea } \\
\text { No } \\
\text { Yes }\end{array}$ & $\begin{array}{c}3(1.8 \%) \\
164(98.2 \%)\end{array}$ & $\begin{array}{c}0(0.0 \%) \\
8(100.0 \%)\end{array}$ & 0.146 & 0.702 \\
\hline $\begin{array}{c}\text { Vomiting } \\
\text { No } \\
\text { Yes }\end{array}$ & $\begin{array}{c}2(1.2 \%) \\
165(98.8 \%)\end{array}$ & $\begin{array}{c}0(0.0 \%) \\
8(100.0 \%)\end{array}$ & 0.097 & 0.756 \\
\hline $\begin{array}{l}\text { Hematemesis } \\
\text { No } \\
\text { Yes }\end{array}$ & $\begin{array}{c}155(92.8 \%) \\
12(7.2 \%)\end{array}$ & $\begin{array}{l}5(62.5 \%) \\
3(37.5 \%)\end{array}$ & 8.952 & $0.003^{*}$ \\
\hline $\begin{array}{l}\text { Abdominal pain } \\
\text { No } \\
\text { Yes } \\
\end{array}$ & $\begin{array}{c}100(59.9 \%) \\
67(40.1 \%) \\
\end{array}$ & $\begin{array}{l}3(37.5 \%) \\
5(62.5 \%)\end{array}$ & 1.579 & 0.209 \\
\hline $\begin{array}{l}\text { Agitation } \\
\text { No } \\
\text { Yes } \\
\end{array}$ & $\begin{array}{c}153(91.6 \%) \\
14(8.4 \%) \\
\end{array}$ & $\begin{array}{l}3(37.5 \%) \\
5(62.5 \%) \\
\end{array}$ & 23.101 & $<0.001 * *$ \\
\hline $\begin{array}{c}\text { Seizures } \\
\text { No } \\
\text { Yes }\end{array}$ & $\begin{aligned} 167 & (100.0 \%) \\
0 & (0.0 \%)\end{aligned}$ & $\begin{array}{l}7(87.5 \%) \\
1(12.5 \%)\end{array}$ & 20.995 & $<0.001 * *$ \\
\hline $\begin{array}{c}\text { Shock } \\
\text { No } \\
\text { Yes }\end{array}$ & $\begin{array}{c}165(98.8 \%) \\
2(1.2 \%)\end{array}$ & $\begin{array}{c}0(0.0 \%) \\
8(100.0 \%)\end{array}$ & 0.097 & 0.756 \\
\hline
\end{tabular}

$\mathrm{n}=$ number; t-Independent Sample t-test; $\mathrm{x} 2$ : Chi-square test $\mathrm{p}$-value $>0.05$ Non significant; *p-value $<0.05$ Significant; ${ }^{* *}$-value $<0.001$ highly significant.

Table (9): Comparison between patients who needed hemodialysis and patients who did not need according to laboratory investigations.

\begin{tabular}{|c|c|c|c|c|}
\hline \multirow[b]{2}{*}{ Parameters } & \multicolumn{2}{|c|}{ Hemodialysis } & \multirow[b]{2}{*}{$x 2 /$ t-test } & \multirow[b]{2}{*}{ p-value } \\
\hline & $\begin{array}{c}\text { No } \\
(n=167)\end{array}$ & $\begin{array}{c}\text { Yes } \\
(\mathrm{n}=8)\end{array}$ & & \\
\hline $\begin{array}{l}\text { Theophylline level }(\boldsymbol{\mu g} / \mathbf{m l}) \\
(\text { Mean } \pm \mathrm{SD})\end{array}$ & $35.49 \pm 22.93$ & $56.7 \pm 30.89$ & 14.838 & $<0.001 * *$ \\
\hline $\begin{array}{c}\text { Serum glucose } \\
\text { Abnormal } \\
\text { Normal } \\
\end{array}$ & $\begin{array}{l}129(77.2 \%) \\
38(22.8 \%) \\
\end{array}$ & $\begin{array}{l}5(62.5 \%) \\
3(37.5 \%) \\
\end{array}$ & 0.282 & 0.595 \\
\hline $\begin{array}{c}\text { Serum } \boldsymbol{K} \\
\text { Abnormal } \\
\text { Normal }\end{array}$ & $\begin{array}{l}108(64.7 \%) \\
59(35.3 \%)\end{array}$ & $\begin{array}{l}5(62.5 \%) \\
3(37.5 \%)\end{array}$ & 0.016 & 0.900 \\
\hline $\begin{array}{ll}\boldsymbol{p H} \\
\text { Abnormal } \\
\text { Normal }\end{array}$ & $\begin{array}{l}79(47.3 \%) \\
88(52.7 \%)\end{array}$ & $\begin{array}{l}5(62.5 \%) \\
3(37.5 \%)\end{array}$ & 0.229 & 0.632 \\
\hline $\begin{array}{l}\mathrm{PCO} 2 \\
\text { Abnormal } \\
\text { Normal } \\
\end{array}$ & $\begin{array}{l}77(46.1 \%) \\
90(53.9 \%) \\
\end{array}$ & $\begin{array}{l}6(75.0 \%) \\
2(25.0 \%) \\
\end{array}$ & 1.530 & 0.216 \\
\hline $\begin{array}{c}\boldsymbol{H C O} 3 \\
\text { Abnormal } \\
\text { Normal } \\
\end{array}$ & $\begin{array}{l}90(53.9 \%) \\
77(46.1 \%) \\
\end{array}$ & $\begin{array}{c}8(100.0 \%) \\
0(0.0 \%) \\
\end{array}$ & 4.847 & $0.028^{*}$ \\
\hline $\begin{array}{l}\boldsymbol{T L C} \\
\text { Abnormal } \\
\text { Normal }\end{array}$ & $\begin{array}{c}137(82.1 \%) \\
30(17.9 \%)\end{array}$ & $\begin{array}{c}8(100.0 \%) \\
0(0.0 \%)\end{array}$ & 4.037 & 0.072 \\
\hline
\end{tabular}

$\mathrm{n}=$ number; SD: standard deviation; TLC: total leucocytic count; t-Independent Sample t-test; $\mathrm{x} 2$ : Chi-square test; $\mathrm{p}$ : value $>0.05$ : non-significant; *p-value $<0.05$ : significant; ${ }^{*}$ p-value $<0.001$ : highly significant. 
Table (10): Comparison between patients who needed hemodialysis and patients who did not need according to vital data.

\begin{tabular}{|c|c|c|c|c|}
\hline \multirow[b]{2}{*}{ Parameters } & \multicolumn{2}{|c|}{ Hemodialysis } & \multirow[b]{2}{*}{$\mathrm{x} 2 / \mathrm{t}$-test } & \multirow[b]{2}{*}{ p-value } \\
\hline & $\begin{array}{c}\text { No } \\
(n=167)\end{array}$ & $\begin{array}{l}\text { Yes } \\
(n=8)\end{array}$ & & \\
\hline $\begin{array}{c}\text { Pulse } \\
\text { Abnormal } \\
\text { Normal }\end{array}$ & $\begin{array}{l}94(56.3 \%) \\
73(43.7 \%)\end{array}$ & $\begin{array}{l}7(87.5 \%) \\
1(12.5 \%)\end{array}$ & 6.328 & $0.036^{*}$ \\
\hline $\begin{array}{c}\text { Blood pressure } \\
\text { Abnormal } \\
\text { Normal } \\
\end{array}$ & $\begin{array}{l}36(21.6 \%) \\
131(78.4 \%) \\
\end{array}$ & $\begin{array}{c}8(100.0 \%) \\
0(0.0 \%)\end{array}$ & 0.028 & 0.867 \\
\hline $\begin{array}{c}\text { Respiratory rate } \\
\text { Abnormal } \\
\text { Normal }\end{array}$ & $\begin{array}{l}92(55.1 \%) \\
75(44.9 \%)\end{array}$ & $\begin{array}{l}6(75.0 \%) \\
2(25.0 \%) \\
\end{array}$ & 5.228 & $0.046^{*}$ \\
\hline $\begin{array}{c}\boldsymbol{E C G} \\
\text { Abnormal } \\
\text { Normal }\end{array}$ & $\begin{array}{l}95(56.9 \%) \\
72(43.1 \%)\end{array}$ & $\begin{array}{l}6(75.0 \%) \\
2(25.0 \%)\end{array}$ & 13.866 & $<0.001 * *$ \\
\hline
\end{tabular}

$\mathrm{n}=$ number; t-Independent Sample t-test; $\mathrm{x} 2$ : Chi-square test; $\mathrm{p}$ : value $>0.05$ : non-significant; ${ }^{*} \mathrm{p}$-value $<0.05$ : significant; ${ }^{* *} \mathrm{p}$-value $<0.001$ : highly significant.

Theophylline poisoning hemodialysis (TPH) score:

From the results of the present study, it was determined that there were 9 important parameters which affect the severity of theophylline poisoning and the need for hemodialysis from which theophylline poisoning hemodialysis (TPH) score was calculated. These parameters were: theophylline level, duration of hospital stay, respiratory rate, pulse, $\mathrm{HCO} 3$ value, presence of hematemesis, seizures, agitation and ECG abnormalities. The best score is 0 , while the worst score is 9 (Table 11).

Table (11): Theophylline poisoning hemodialysis (TPH) score

\begin{tabular}{|c|c|}
\hline Parameter & Score \\
\hline $\begin{array}{c}\text { Hematemesis } \\
\text { Absent } \\
\text { Present } \\
\end{array}$ & $\begin{array}{l}0 \\
1 \\
\end{array}$ \\
\hline $\begin{array}{c}\text { Agitation } \\
\text { Absent } \\
\text { Present }\end{array}$ & $\begin{array}{l}0 \\
1\end{array}$ \\
\hline $\begin{array}{c}\text { Seizures } \\
\text { Absent } \\
\text { Present }\end{array}$ & $\begin{array}{l}0 \\
1\end{array}$ \\
\hline $\begin{aligned} \text { Pulse } & \\
& <110 \mathrm{beat} / \mathrm{min} . \\
& >110 \mathrm{beat} / \mathrm{min} .\end{aligned}$ & $\begin{array}{l}0 \\
1\end{array}$ \\
\hline $\begin{array}{l}\text { Respiratory rate } \\
<27 \text { breath } / \mathrm{min} . \\
>27 \mathrm{breath} / \mathrm{min} .\end{array}$ & $\begin{array}{l}0 \\
1\end{array}$ \\
\hline $\begin{array}{l}\text { HCO3 level } \\
>23 \mathrm{mmol} / \mathrm{l} \\
<23 \mathrm{mmol} / \mathrm{l}\end{array}$ & $\begin{array}{l}0 \\
1\end{array}$ \\
\hline $\begin{array}{r}\text { Theophylline level } \\
<56.7 \mu \mathrm{g} / \mathrm{ml} \\
>56.7 \mu \mathrm{g} / \mathrm{ml}\end{array}$ & $\begin{array}{l}0 \\
1\end{array}$ \\
\hline $\begin{array}{ll}\text { ECG } & \\
& \text { Normal } \\
& \text { Abnormal } \\
\end{array}$ & $\begin{array}{l}0 \\
1\end{array}$ \\
\hline $\begin{array}{c}\text { Duration of hospital stay } \\
<2 \text { days } \\
>2 \text { days }\end{array}$ & $\begin{array}{l}0 \\
1\end{array}$ \\
\hline Best score & 0 \\
\hline Worst score & 9 \\
\hline
\end{tabular}


Table (12) shows the total theophylline poisoning hemodialysis (TPH) score in cases who needed hemodialysis and cases who did not. There was a highly significant difference $(p<0.001)$ between them according to mean value of TPH score as confirmed by Z-test. The mean TPH score in cases needed hemodialysis was $5.14 \pm 1.85$ ranged from 2-9 while in nonhemodialysis cases it was $1.87 \pm 1.19$ ranged from 0 to 6 .

Table (12): $Z$ test statistical analysis for comparison between theophylline intoxicated patients who needed hemodialysis and patients who did not according to total theophylline poisoning hemodialysis $(\mathrm{TPH})$ score.

\begin{tabular}{|l|c|c|c|c|}
\hline \multirow{2}{*}{ Total TPH Score } & \multicolumn{2}{|c|}{ Hemodialysis } & \multirow{2}{*}{ z-test } & \multirow{2}{*}{ p-value } \\
\cline { 2 - 3 } & No (n=167) & Yes $(\mathbf{n}=\mathbf{8})$ & & \multirow{2}{*}{33.120} \\
\hline Mean \pm SD & $1.87 \pm 1.19$ & $5.14 \pm 1.85$ & \multirow{2}{*}{$<0.001^{* *}$} \\
\hline Range & $0-6$ & $2-9$ & & \\
\hline
\end{tabular}

$\mathrm{n}=$ number; TPH: Theophylline poisoning hemodialysis, SD: standard deviation; $\mathrm{p}$ : value $>0.05$ : non-significant; ${ }^{*} \mathrm{p}$-value $<0.05$ : significant; **p-value $<0.001$ : highly significant

Receiver operating characteristics (ROC) curve was used to define the best cut off value of total TPH score which was $\geq 5$, with sensitivity of $87.5 \%$ specificity of $79.6 \%$, positive predictive value of $71.1 \%$ and negative predictive value of $99.3 \%$ with diagnostic accuracy of $80 \%$. Finally, cases with total TPH score greater than or equal 5 had a higher probability for hemodialysis (Table 13 and Figure 1).

Table (13): The best Cut-off of total theophylline poisoning hemodialysis (TPH) score for prediction of hemodialysis in theophylline intoxicated patients.

\begin{tabular}{|l|c|c|c|c|c|c|}
\hline & Cut-off & Sensitivity & Specificity & PPV & NPV & Accuracy \\
\hline TPH score & $\geq 5$ & $87.5 \%$ & $79.6 \%$ & $71.1 \%$ & $99.3 \%$ & $80 \%$ \\
\hline
\end{tabular}

TPH: Theophylline poisoning hemodialysis, PPV: Positive predictive value, NPV: negative predictive value.

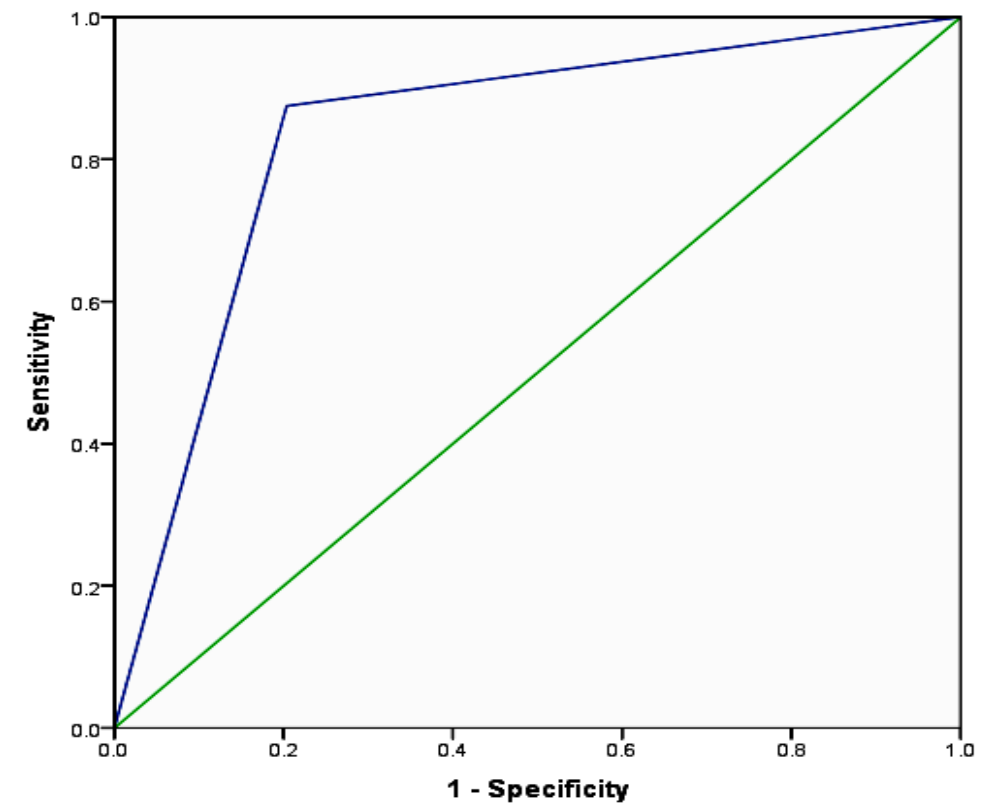

Fig. (1): Receiver-operating characteristic (ROC) curve for prediction of hemodialysis using Theophylline poisoning hemodialysis (TPH) score. 


\section{Discussion}

Theophylline is a methylxanthine prescribed in patients with moderate to severe asthma (Greene et al., 2018). Toxic symptoms appear at a plasma concentration over $20 \mu \mathrm{g} / \mathrm{ml}$ which is the needed therapeutic range (Yaman et al., 2016) as the British National Formulary (BNF) suggested target theophylline plasma concentrations of $10-20 \mathrm{mg} / \mathrm{l}$ (Hopkins and MacKenzie-Ross, 2016).

Side effects of theophylline could be severe and even cause death and early recognition of severity of theophylline toxicity can be lifesaving and aggressive supportive care is the key to improve clinical outcomes (Aggelopoulou et al., 2018). So, this study aimed to evaluate theophylline toxic manifestations to construct a predictive score to identify those who are at risk and require hemodialysis to improve management of acute theophylline intoxicated patients as early diagnosis and appropriate treatment is often lifesaving.

All patients of this study intentionally ingested theophylline as a suicidal attempt; most of them were females in their $20 \mathrm{~s}$. The mean delayed time was $7.29 \pm 5.08$ hours. The mean duration of hospital stay was $1.57 \pm 0.72$ days. No cases needed ventilator support. All cases were discharged with no deaths. Kapoor et al. (2015) stated that theophylline use has been associated with an increase of suicidal ideation in asthma patients. The main risk factors for suicide attempts by poisoning were female sex, aged 15-40 years (PIRES, 2014).

According to the American Association of Poison Control Centers, there were 1641 exposures to theophylline ten of them died in 1999 (Litovitz et al., 2001) and by 2014, the total number of cases involving theophylline had decreased to 199, with two deaths and eight major effects (Mowry et al., 2015).
The mean theophylline level for the patients of the present study was $40.38 \pm 25.30$ ranged from 15-90.8 $\mu \mathrm{g} / \mathrm{mL}$. Aggelopoulou et al. (2018) reported that minor but frequent manifestations occurred at theophylline concentrations $(80-100 \mu \mathrm{g} / \mathrm{mL})$ while in chronic exposure, the levels could be lower $(40-60 \mu \mathrm{g} / \mathrm{mL})$.

In the current study, eight cases $(4.6 \%)$ needed hemodialysis. This was supported by Yaman et al. (2016) who reported that continuous veno-venous hemodialysis (CVVHD) is considered only in critically ill patients with severe theophylline poisoning.

In the current study the commonest symptoms of theophylline poisoned cases were nausea and vomiting followed by abdominal pain and agitation. Hematemesis was found in $8.6 \%$ of the cases. Two of the patients were shocked and only one patient had seizures. Kapoor et al. (2015) supported these results as they stated that patients with theophylline overdose could have nausea, vomiting, abdominal pain, coarse muscle tremors and hypotension.

Also, Greene et al. (2018) reported that gastrointestinal signs and symptoms are common in acute theophylline toxicity and occasionally are associated with hematemesis while neurological manifestations may include tremor, irritability, lethargy and seizures.

All cases of the present study had high TLC, most of them were hyperglycemic, $64.6 \%$ of the cases were hypokalemic and the blood $\mathrm{PH}$ was acidic in $66.7 \%$ of the cases. These were in agreement with the findings of Greene et al. (2018) and supported by Kapoor et al. (2015) and Aggelopoulou et al. (2018) who reported that hypokalemia, hypercalcemia, hyperglycemia, and acidosis are common metabolic disturbances after acute theophylline overdose. 
Greene et al. (2018) attributed hypokalemia to either transcellular shift or gastrointestinal loss while hyperglycemia resulted from increased catecholamine activity. Metabolic acidosis is due to elevated lactic acid either from tissue hypoperfusion or muscular hyperactivity.

Theophylline has profound cardiotoxicity so its use is limited nowadays (Aggelopoulou et al. 2018). This statement was in accordance with the results of the current study as most of cases were hypertensive, tachycardiac and had abnormal ECG findings. The most common ECG abnormality was sinus tachycardia followed by prolonged QTc interval and abnormal $\mathrm{T}$ wave. These results were in agreement with those of Greene et al. (2018) who found tachycardia was the commonest cardiovascular manifestation among their patients and they explained it due to increase catecholamine concentrations which lead to cardiac arrhythmias.

Kapoor et al. (2015) stated that theophylline in toxic doses leads to cardiac arrhythmias like atrial fibrillation, ventricular arrhythmias and seizures.

All studied patients were tachypneic. This result was supported by Hopkins and MacKenzie-Ross (2016) who reported that theophylline has the ability to stimulate the central respiratory drive; leading to deep and rapid breathing that antagonize all adenosine receptor types and increased hormone release as norepinephrine (Aggelopoulou et al., 2018).

Yaman et al. (2016) stated that the consequences of severe theophylline toxicity are seizures, cardiac arrhythmias, and death.

From the results of the present study theophylline poisoning hemodialysis (TPH) score was constructed to find out the probability of patient's need for hemodialysis. It consisted of nine important parameters which were: theophylline level $>56.7 \mu \mathrm{g} / \mathrm{ml}$, time of stay $>2$ days, pulse $>110$ beat $/ \mathrm{min}$, respiratory rate $>27$ breath $/ \mathrm{min}, \mathrm{HCO} 3<23 \mathrm{mmol} / 1$, presence of hematemesis, seizures, agitation, and abnormal ECG findings. Finally, patients with total TPH score equal greater than 5 (cases presented with 5 or more of abnormal values of the previous 9 parameters) had a high probability for hemodialysis.

While Ghannoum et al. (2015) concluded that intermittent dialysis following acute theophylline overdose is recommended in specific circumstances; when theophylline level $>100 \mathrm{mg} / \mathrm{L}$, presence of seizures, shock, life-threatening dysrhythmia and rising theophylline level or clinical deterioration despite optimal care.

\section{Conclusions}

Theophylline toxicity could be lifethreatening and may require hemodialysis as a lifesaving measure. The current study constructs a new score for prediction of the probability of need of acute theophylline intoxicated patient for hemodialysis from clinical and laboratory results. It consists of nine important parameters which are theophylline level $>56.7 \mu \mathrm{g} / \mathrm{ml}$, time of hospital stay $>2$ days, pulse $>110$ beat $/ \mathrm{min}$, respiratory rate $>27$ breath $/ \mathrm{min}, \mathrm{HCO} 3<23$ mmol, presence of hematemesis, seizures, agitation, and abnormal ECG findings. Theophylline intoxicated patients with TPH score greater than or equal to 5 had a higher probability for hemodialysis.

\section{Recommendation}

The current study recommended that clinicians in emergency rooms should use theophylline poisoning hemodialysis (TPH) score for all patients with acute theophylline toxicity to identify who is at risk and needs hemodialysis for better outcome. 


\section{References}

Aggelopoulou, E.; Tzortzis, S.; Tsiourantani F.; et al. (2018): "Atrial fibrillation and shock: Unmasking theophylline toxicity". Medical Principal and Practice, 27(4): 387-391.

Barnes, P.J. (2013): “Theophylline”. Am. J. Resp. Crit. Care Med., 188:901-906.

Ghannoum, M.; Wiegand, T.J.; Liu, K.D. et al. (2015):" Extracorporeal treatment for theophylline poisoning: Systematic review and recommendations from the EXTRIP workgroup". Clinical Toxicology Journal, 53(4): 215-229.

Greene, S.C.; Halmer, T.; Carey, J.M.; et al. (2018): "Theophylline toxicity: An old poisoning for a new generation of physicians". Turkish Journal of Emergency Medicine, 18(1): 37-39.

Hopkins, M.E. and MacKenzie-Ross, R.V. (2016): "Case report: The risks associated with chronic theophylline therapy and measures designed to improve monitoring and management". BMC Pharmacology and Toxicology, 17: 13.

Horita, N.; Miyazawa, N.; Kojima, R; et al. (2016): "Chronic use of theophylline and mortality in chronic obstructive pulmonary disease: A meta-analysis". Arch Bronconeumol., 52(5):233-238.

Kapoor, S.; Thakkar, J. and Aggarwal, V. (2015): "Theophylline toxicity leading to suicidal ideation in a patient with no prior psychiatric illness". SAGE Open Medical Case Report., 3: doi: 10.1177/2050313X15583208

Litovitz, T.L.; Klein-Schwartz, W. and White S. (2001): "1999 annual report of the American Association of Poison Control Centers toxic exposure surveillance system". American Journal of Emergency Medecine, 18(5):517-574.

McGrath, J.L. and Bachmann, D.J. (2018): Vital signs measurements. In: Roberts and Hedges'Clinical Procedures in Emergency Medicine E-Book Roberts JR, Hedges JR (eds),7th ed., Elsevier Health Sciences, P.P.1-24.

Mowry, J.B.; Spyker, D.A. and Brooks, D.E. (2015): "Annual report of the American Association of Poison Control Centers' national Poison data system (NPDS): 32nd annual report". Clinical Toxicology, 53:962-1147.

PIRES, M.C.C. (2014): "Risk factors of suicide attempts by poisoning: review". SciELO., 36(2):63-74.

Postema, P.G. and Wilde, A.A. (2014): "The measurement of the QT interval". Current Cardiology Review, 10(3):287-294.

Sheehan, M. and Haythorn, P. (1976): "Rapid gas chromatographic determination of underivatized theophylline in whole blood". Journal of Chromatography, 117:392-398.

Wu, J.P.; Wu, Q.; Sun, X; et al. (2013): "Corticosteroid resistance in chronic obstructive pulmonary disease: new uses of theophylline". Chinese Medical Journal, 126: 965- 970.

Yaman, A.; Kendirli, T.; Ödek, Ç; et al. (2016): "Severe theophylline poisoning treated with continuous venovenous hemodialysis in a child". Turkish Journal of Pediatrics, 58(3):297-300. 


\section{بناي مقياس للتنبؤ بالغسيل الكلوى في مرضى التسمم الحاد بعقار الثيوفيلين}

\section{وسام عبدالسلام عبدالوهابا ورانيا حسين محمد حسين r P}

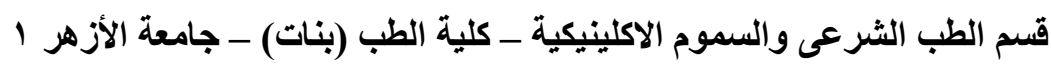

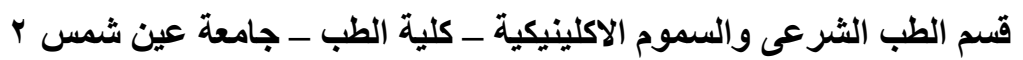

قد يكون التسمم بعقار الثيوفيلين مهدد للحياة، وهذا بسبب مـا يسببه من انخفاض ضغط الدم الحاد ، و اضـطر اب شـديد فـى ضـربات القلب أو التشنجات والتـى قـد تتطلب از الـة الثيـوفيللين عـن طريـق الغسيل الكلوى. هدفت هذه الدر اسـة إلى تقيم الأعر اض السامة للثيوفيلين لبنـاء مقيـس تنبؤى لتحديد المرضىى الذين يتعرضون للخطر ويحتاجون إلى الغسيل الكلوى.

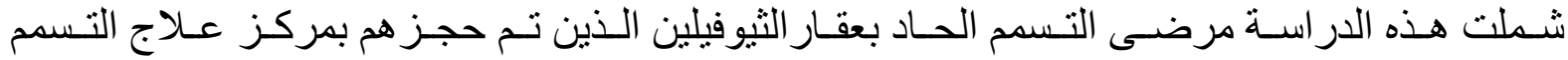
بمستشفيات جامعة عين شمس خلال فترة ستة أشهر من بدايـة شهر يناير 9 1 ـ إلى نهايـة شهر يونيو 9 1 ـ ب.

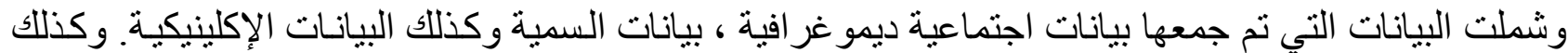
تم تسجيل مصير المرضـى واحتيـاجهم للغسيل الكلوى. وقد تم عمل الفحوصـات النمطيـة وقياس مستوى عقار الثيوفيللين بالدم لكل مريض بالإضافة إلى تسجيلات رسم القلب الكهربائى .

تضمنت هذه الدر اسة ال مريض بالتسمم الحاد بعقار الثيوفيلين و التى انطبقت عليهم شروط الدر اسـة.

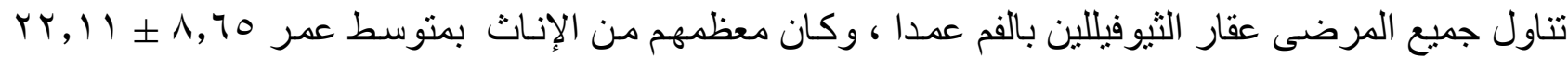

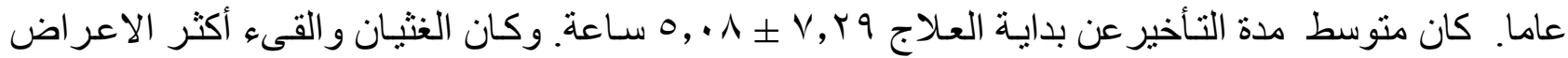

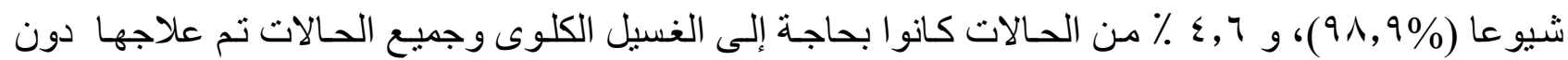

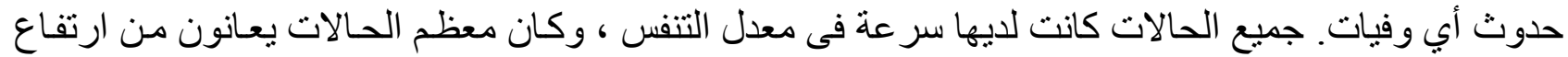
فى ضغط الدم و سر عة فى ضربات القلب ونتائج غير طبيعية لرسم القلب الكهربائى . خلصت هذه الدر اسـة الى بنـاء مقيـاس الغسيل الكلوى لمرضـى التسمم بعقار الثيوفيللين لتحديد احتماليـة

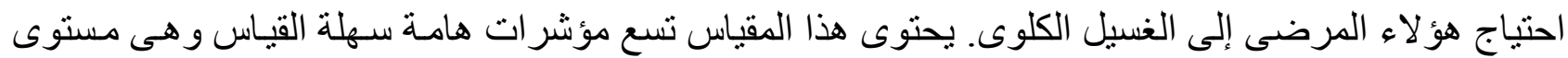

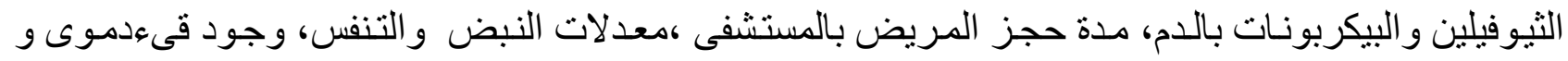

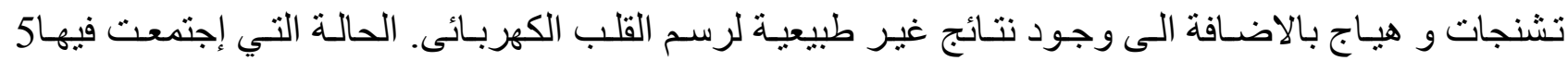
تغير ات فأكثر تعد حالة حرجة و هنالك احتمال كبير لاحتياجها إلى عمل غسيل كلوى. 